\title{
Efficacy and safety of febuxostat in the treatment of hyperuricemia in stable kidney transplant recipients
}

This article was published in the following Dove Press journal:

Drug Design, Development and Therapy

17 February 2014

Number of times this article has been viewed

\author{
Tadashi Sofue' \\ Masashi Inui² \\ Taiga Hara' \\ Yoko Nishijima' \\ Kumiko Moriwaki' \\ Yushi Hayashida ${ }^{3}$ \\ Nobufumi Ueda ${ }^{3}$ \\ Akira Nishiyama ${ }^{4}$ \\ Yoshiyuki Kakehi ${ }^{3}$ \\ Masakazu Kohno' \\ 'Division of Nephrology and Dialysis, \\ Department of Cardiorenal and \\ Cerebrovascular Medicine, Kagawa \\ University, Kagawa, ${ }^{2}$ Department of \\ Urology, Tokyo Women's Medical \\ University, Tokyo, ${ }^{3}$ Department \\ of Urology, ${ }^{4}$ Department of \\ Pharmacology, Kagawa University, \\ Kagawa, Japan
}

Correspondence: Tadashi Sofue Department of Cardiorenal and Cerebrovascular Medicine, Faculty of Medicine, Kagawa University, I750-I Ikenobe, Miki-cho, Kita-gun, Kagawa 76I-0793, Japan

Tel +8I 8 789| 2I 49 ext 2623

Fax +81878912149

Email sofue@med.kagawa-u.ac.jp
Background: Post-transplant hyperuricemia (PTHU), defined as serum uric acid concentration $\geq 7.0 \mathrm{mg} / \mathrm{dL}$ or need for treatment with allopurinol or benzbromarone, reduces long-term allograft survival in kidney transplant recipients. Febuxostat, a new nonpurine selective xanthine oxidase inhibitor, is well tolerated in patients with moderate renal impairment. However, its efficacy and safety in kidney recipients with PTHU is unclear. We therefore assessed the efficacy and safety of febuxostat in stable kidney transplant recipients with PTHU.

Methods: Of 93 stable adult kidney transplant recipients, 51 were diagnosed with PTHU (PTHU group) and 42 were not (NPTHU group). Of the 51 patients with PTHU, 26 were treated with febuxostat (FX group) and 25 were not (NFX group), at the discretion of each attending physician. One-year changes in serum uric acid concentrations, rates of achievement of target uric acid $(<6.0 \mathrm{mg} / \mathrm{dL})$, estimated glomerular filtration rates in allografts, and adverse events were retrospectively analyzed in the FX, NFX, and NPTHU groups.

Results: The FX group showed significantly greater decreases in serum uric acid $(-2.0 \pm 1.1 \mathrm{mg} / \mathrm{dL}$ versus $0.0 \pm 0.8 \mathrm{mg} / \mathrm{dL}$ per year, $P<0.01)$ and tended to show a higher rate of achieving target uric acid levels ( $50 \%$ versus $24 \%$; odds ratio 3.17 [95\% confidence interval $0.96-10.5$ ], $P=0.08$ ) than the NFX group. Although baseline allograft estimated glomerular filtration rates tended to be lower in the FX group than in the NFX group $\left(40 \pm 14 \mathrm{~mL} / \mathrm{min} / 1.73 \mathrm{~m}^{2}\right.$ versus $47 \pm 19 \mathrm{~mL} / \mathrm{min} / 1.73 \mathrm{~m}^{2}$ ), changes in allograft estimated glomerular filtration rate were similar $\left(+1.0 \pm 4.9 \mathrm{~mL} / \mathrm{min} / 1.73 \mathrm{~m}^{2}\right.$ versus $-0.2 \pm 6.9 \mathrm{~mL} / \mathrm{min} / 1.73 \mathrm{~m}^{2}$ per year, $\left.P=0.50\right)$. None of the patients in the FX group experienced any severe adverse effects, such as pancytopenia or attacks of gout, throughout the entire study period. Nephrologists were more likely than urologists to start febuxostat in kidney transplant recipients with PTHU (69\% versus $8 \%$ ).

Conclusion: Treatment with febuxostat sufficiently lowered uric acid levels without severe adverse effects in stable kidney transplant recipients with PTHU.

Keywords: post-transplant hyperuricemia, febuxostat, uric acid, chronic kidney disease

\section{Introduction}

Many kidney transplant recipients experience post-transplant hyperuricemia (PTHU). ${ }^{1,2}$ Several factors were reported to predispose recipients to hyperuricemia, including insufficient allograft function, ${ }^{3}$ treatment with diuretics, immunosuppression with cyclosporine, ${ }^{4}$ a calcineurin inhibitor (CNI), and immunosuppression with mizoribine. ${ }^{5}$ In the general population, asymptomatic hyperuricemia is a risk factor for the onset and progression of chronic kidney disease and for cardiovascular disease. ${ }^{6,7}$ Similarly, PTHU is associated with allograft loss and onset of cardiovascular disease in kidney transplant recipients. ${ }^{3,8-11}$ Conventional uric acid-lowering therapy consists principally of allopurinol 
and benzbromarone. Allopurinol inhibits the production of uric acid by inhibiting xanthine oxidase. However, both allopurinol and its active metabolite oxypurinol are excreted in the urine. ${ }^{12}$ Therefore, kidney transplant recipients with chronic kidney disease cannot be administered doses of allopurinol sufficient to lower uric acid. In contrast, benzbromarone increases the urinary excretion of uric acid, but its effects are reduced in transplant recipients with chronic kidney disease, in whom it is difficult achieve target uric acid levels using conventional uric acid-lowering therapy. It has not yet been determined whether uric acid-lowering therapy has beneficial effects on allograft survival rate and the onset of cardiovascular disease in kidney transplant recipients with PTHU.

Febuxostat, a new nonpurine selective xanthine oxidase inhibitor, is well tolerated in patients with gout and in those with mild or moderate renal impairment. ${ }^{13-15}$ However, the efficacy and safety of febuxostat in stable kidney transplant recipients with asymptomatic hyperuricemia has not been assessed. We therefore investigated the efficacy and safety of febuxostat in kidney transplant recipients with PTHU.

\section{Patients and methods Subjects}

We retrospectively analyzed 93 adult kidney transplant recipients who visited Kagawa University Hospital between June 2012 and June 2013. Recipients who were treated with azathioprine and those who underwent kidney transplantation between June 2011 and June 2013 were excluded. The protocols, patient information, and informed consent forms were reviewed and approved by the ethics committee of Kagawa University (H25-052).

\section{Study design}

The 93 kidney transplant recipients were treated by four nephrologists and two urologists. PTHU was defined as uric acid $\geq 7.0 \mathrm{mg} / \mathrm{dL}$ ( $\geq 0.42 \mathrm{mmol} / \mathrm{L}$ ) or need for treatment with the conventional uric acid-lowering drugs, allopurinol and benzbromarone. The decision to start treatment with febuxostat was at the discretion of each attending physician.

Of the 93 kidney transplant recipients, 51 were diagnosed with PTHU (PTHU group) and 42 were not (NPTHU group) in June 2012. Of the 51 recipients with PTHU, 26 received febuxostat (FX group) and 25 did not (NFX).

The 26 patients in the FX group were started on febuxostat treatment between May and September 2012. These 26 patients were divided into two subgroups, ie, those who had been treated with conventional uric acid-lowering therapy before treatment with febuxostat (FC subgroup, $\mathrm{n}=11$ ) and those who had not (FN subgroup, $n=15$ ). Of the eleven patients in the FC subgroup, five had received allopurinol (100 mg/day) and six had received benzbromarone (two receiving $25 \mathrm{mg} /$ day and four receiving $50 \mathrm{mg} /$ day). Treatment with febuxostat was started at $10 \mathrm{mg} /$ day and increased to $20 \mathrm{mg} /$ day if serum uric acid levels remained over $7 \mathrm{mg} / \mathrm{dL}$. Of the 26 patients in the FX group, 17 (65\%) were finally treated with febuxostat $20 \mathrm{mg} /$ day.

The 25 patients in the NFX group were also divided into two subgroups, ie, those who had been under observation without intervention for PTHU (OB group, n=12) and those who were being continued on conventional uric acidlowering therapy (CT group, $n=13$ ). Of the 13 patients in the CT group, six had received allopurinol (100 mg/day) and seven had received benzbromarone (four receiving $25 \mathrm{mg} /$ day and three receiving $50 \mathrm{mg} /$ day). The type of conventional uric acid-lowering therapy was not switched during the study.

Study entry was defined as the time of starting treatment with febuxostat in the FX group and as June 2012 in the NFX and NPTHU groups. Serum uric acid concentrations were determined at baseline and at one, 3, 6, and 12 months after study entry. One-year changes in serum uric acid (mg/dL per year) were calculated as the concentration at 12 months minus the concentration at baseline. The target uric acid level was set at $<6.0 \mathrm{mg} / \mathrm{dL}(<0.36 \mathrm{mmol} / \mathrm{L}) .{ }^{16}$ The rate of achievement of the target uric acid level (\%) was calculated as the number of patients who achieved the target uric acid level divided by the number of patients in that group, and was evaluated at baseline and after 12 months. Rates of achievement of target uric acid levels at baseline and after 12 months were compared individually in the FX and NFX groups, with between-group differences assessed after 12 months.

Allograft function was based on the estimated glomerular filtration rate (eGFR), which was calculated using the Modification of Diet in Renal Disease study equation modified for Japanese individuals. ${ }^{17-19}$ eGFR was measured at baseline and at one and 12 months after entry, with one-year changes in eGFR $\left(\mathrm{mL} / \mathrm{min} / 1.73 \mathrm{~m}^{2}\right.$ per year) calculated as the eGFR at 12 months minus the eGFR at baseline. Blood pressure, cell counts (including white blood cells and platelets), hemoglobin concentration, and liver function tests, including serum alanine aminotransferase, aspartate aminotransferase, alkaline phosphatase, and total bilirubin concentrations, were measured at baseline and after 12 months. eGFR and blood pressure were compared in the FX and NFX groups. Cell counts, hemoglobin concentration, and liver function were compared in the FX and NFX + NPTHU groups. Severe adverse effects, 
cardiovascular events, and allograft loss were monitored throughout the study period.

Maintenance immunosuppressive regimens were based principally on combinations of three drugs, ie, tacrolimus or cyclosporine, mycophenolate mofetil or mizoribine, and methylprednisolone. Types of immunosuppressive agents were not switched during the study. Concomitant drugs, including angiotensin II type 1 receptor blockers, calcium channel blockers, proton pump inhibitors, statins and erythropoiesis-stimulating agents, were also checked at baseline. Blood tacrolimus concentrations were determined using a microparticle enzyme immunoassay (IMx ${ }^{\mathrm{TM}}$; Abbott Laboratories, Abbott Park, IL, USA) ${ }^{20}$ The dose-normalized tacrolimus blood concentration ( $\mathrm{ng} \mathrm{mL} \mathrm{mL}^{-1} / 0.1 \mathrm{mg} \mathrm{kg}^{-1}$ ) ${ }^{20}$ was evaluated at baseline and at 12 months after entry and was calculated using the formula: trough tacrolimus blood concentration $(\mathrm{ng} / \mathrm{mL})$ divided by body weight-normalized tacrolimus dose $(0.1 \mathrm{mg} / \mathrm{kg})$, with the latter calculated as $0.1 \times$ tacrolimus dosage $(\mathrm{mg}) /$ body weight $(\mathrm{kg})$. To clarify the differences in practice patterns between nephrologists and urologists, the percentages of each starting febuxostat for recipients with PTHU were compared.

\section{Statistical analysis}

All analyses were performed using Statistical Package for the Social Sciences software version 20.0 for Windows (IBM Japan, Tokyo, Japan). Values are presented as the mean \pm standard deviation or number (\%). Categorical variables were compared using the $\chi^{2}$ test and continuous variables using the Student's $t$-test or one-way analysis of variance. A $P$ value $<0.05$ was considered to be statistically significant.

\section{Results \\ Clinical characteristics of the FN, FC, OB, CT, and NPTHU groups}

The characteristics of the 93 kidney transplant recipients are shown in Table 1. Baseline uric acid levels were higher in the FN, FC, and $\mathrm{OB}$ groups than in the CT and NPTHU groups. At baseline, smaller percentages of patients in the FN, FC, and OB groups than in the CT and NPTHU groups had achieved target uric acid levels. The percentage of male recipients tended to be higher in the PTHU group than in the NPTHU group. Baseline eGFR tended to be lower in the FN, FC, and CT groups than in the OB and NPTHU groups.

Table I Recipient characteristics

\begin{tabular}{|c|c|c|c|c|c|c|}
\hline & \multicolumn{2}{|l|}{ PTHU } & \multicolumn{2}{|l|}{$\mathbf{F X}$} & \multirow{2}{*}{$\frac{\text { NFX }}{\text { NPTHU }}$} & \multirow[t]{2}{*}{$P$-value } \\
\hline & $\mathbf{F N}$ & FC & OB & $\mathbf{C T}$ & & \\
\hline Recipients, $\mathrm{n}$ & 15 & 11 & 12 & 13 & 42 & \\
\hline Baseline uric acid (mg/g Cr) & $8.4 \pm 1.0^{*+\dagger}$ & $7.5 \pm 1.5^{*, \dagger}$ & $7.7 \pm 0.6 *, \dagger$ & $6.3 \pm 1.2$ & $5.6 \pm 1.0$ & 0.02 \\
\hline Achievement of target uric acid, n (\%) & 0 & $2(18 \%)$ & 0 & $4(3 \mid \%)$ & $24(57 \%)$ & $<0.01$ \\
\hline Age (years) & $52 \pm 12$ & $44 \pm 10$ & $51 \pm 18$ & $57 \pm 9$ & $49 \pm 14$ & 0.21 \\
\hline Male, n (\%) & $13(87 \%)$ & 10 (91\%) & $9(82 \%)$ & II (85\%) & $24(57 \%)$ & 0.055 \\
\hline Diabetes, n (\%) & $4(27 \%)$ & I (9\%) & $3(25 \%)$ & I (8\%) & 12 (29\%) & 0.43 \\
\hline Baseline eGFR $\left(\mathrm{mL} / \mathrm{min} / \mathrm{l} .73 \mathrm{~m}^{2}\right)$ & $43.2 \pm 13.1$ & $36.7 \pm 15.2$ & $52.7 \pm 24.8$ & $41.3 \pm 11.3$ & $50.8 \pm \mid 5.1$ & 0.03 \\
\hline BMI $\left(\mathrm{kg} / \mathrm{m}^{2}\right)$ & $22.2 \pm 5.0$ & $22.6 \pm 4.7$ & $20.2 \pm 2.3$ & $22.1 \pm 3.7$ & $22.5 \pm 3.5$ & 0.53 \\
\hline Systolic blood pressure (mmHg) & $117 \pm 7$ & $119 \pm 11$ & $121 \pm 13$ & $128 \pm 13$ & $119 \pm 11$ & 0.30 \\
\hline Time after transplantation (years) & $5.8 \pm 3.2$ & $7.1 \pm 4.5$ & $6.8 \pm 3.8$ & $5.8 \pm 3.1$ & $5.0 \pm 3.4$ & 0.33 \\
\hline Cadaver donor, n (\%) & 0 & 0 & I (8\%) & I (8\%) & I (2\%) & 0.59 \\
\hline Blood type incompatible, n (\%) & $4(27 \%)$ & $5(45 \%)$ & $4(33 \%)$ & $6(46 \%)$ & $12(29 \%)$ & 0.66 \\
\hline Tacrolimus-based IS, n (\%) & $12(87 \%)$ & $8(73 \%)$ & $9(75 \%)$ & $9(8 \mid \%)$ & $38(90 \%)$ & 0.51 \\
\hline Trough level of tacrolimus (ng/mL) & $5.0 \pm 1.0$ & $5.4 \pm 1.5$ & $5.4 \pm 1.2$ & $5.0 \pm 1.2$ & $5.6 \pm 1.3$ & 0.47 \\
\hline MMF-based IS, n (\%) & $13(87 \%)$ & $8(64 \%)$ & II (92\%) & 9 (82\%) & 40 (95\%) & 0.38 \\
\hline MMF dose (mg) & $\mathrm{I}, 173 \pm 277$ & $1,000 \pm 289$ & $932 \pm 162$ & $\mathrm{I}, 023 \pm 284$ & $I, 08 I \pm 5 \mid$ & 0.38 \\
\hline mPSL dose (mg) & $2.2 \pm 1.1$ & $2.7 \pm 1.0$ & $2.6 \pm 2.6$ & $2.2 \pm 0.6$ & $2.0 \pm 0.9$ & 0.45 \\
\hline ARB treatment, n (\%) & II $(73 \%)^{*}$ & II $(100 \%)^{*}$ & $8(67 \%)$ & $9(69 \%)$ & $17(40 \%)$ & $<0.01$ \\
\hline CCB treatment, $\mathrm{n}(\%)$ & $7(47 \%)$ & $8(73 \%)^{*}$ & $7(58 \%)^{*}$ & $9(69 \%)^{*}$ & $10(24 \%)$ & $<0.01$ \\
\hline PPI treatment, n (\%) & II (73\%) & $8(73 \%)$ & $7(58 \%)$ & II (85\%) & $33(79 \%)$ & 0.60 \\
\hline Statin treatment, n (\%) & I (7\%) & $5(45 \%)^{*}$ & $4(33 \%)$ & $3(23 \%)$ & $4(10 \%)$ & 0.03 \\
\hline ESA treatment, n (\%) & $3(20 \%)^{*}$ & $2(18 \%)^{*}$ & 0 & $3(23 \%)^{*}$ & 0 & 0.02 \\
\hline
\end{tabular}

Notes: Values are shown as the mean \pm standard deviation or $n(\%) . * P<0.05$ versus NPTHU; ${ }^{\dagger} P<0.05$ versus CT.

Abbreviations: $\mathrm{Cr}$, creatinine; PTHU, post-transplant hyperuricemia; NPTHU, non-post-transplant hyperuricemia; FX, febuxostat group; NFX, non-febuxostat group; FN, newly treated with febuxostat; FC, conversion to febuxostat; OB, observation without intervention; CT, continued conventional therapy; eGFR, estimated glomerular filtration rate; BMI, body mass index; IS, immunosuppressant; MMF, mycophenolate mofetil; mPSL, methylprednisolone; ARB, angiotensin II type I receptor blocker; CCB, calcium channel blocker; PPI, proton pump inhibitor; ESA, erythropoiesis-stimulating agent. 
There were no significant differences between these groups with regard to age, body mass index, baseline blood pressure, and dose of immunosuppressants. Although rates of administration of angiotensin II type 1 receptor blockers, calcium channel blockers, and erythropoiesis-stimulating agents tended to be higher in the PTHU than in the NPTHU group, there were no significant differences between the FX and NFX groups. Background immunosuppressive drug regimens are shown in Table 2. The rates of treatment with immunosuppressive drugs were higher in the PTHU $(\mathrm{FX}+\mathrm{NFX})$ group than in the NPTHU group, including rates of treatment with mizoribine $(24 \%$ versus $2 \%$; odds ratio $13.2,95 \%$ confidence interval $[\mathrm{CI}] 1.61-109, P<0.01)$ and regimens including cyclosporine $(26 \%$ versus $7 \%$; odds ratio 4.33, 95\% CI 1.14-16.5, $P=0.03$ ). However, there were no differences in background immunosuppressive drug regimens between the FX and NFX groups.

\section{Effects of febuxostat on serum uric acid levels}

The effects of febuxostat on serum uric acid levels are shown in Table 3 and Figure 1. Following treatment with febuxostat, serum uric acid levels were lower in the FN and FC groups than in the OB group (Table 3). The oneyear change in serum uric acid levels was significantly greater in the FX group than in the NFX group (Figure 1A). Rates of achievement of target uric acid concentration $(<6.0 \mathrm{mg} / \mathrm{dL})$ are shown in Figure 1B. In the FX group, this rate was significantly higher after one year of treatment with febuxostat than at baseline (odds ratio 12.0, 95\% CI 2.34-61.6, $P<0.01)$. Twelve months after study entry, the rate of achievement of target uric acid levels tended to be higher in the FX group than in the NFX group, but the difference was not statistically significant (odds ratio 3.17, 95\% CI 0.96-10.5, $P=0.08$ ).

Table 2 Background immunosuppressive drug regimens

\begin{tabular}{lllll}
\hline & FX & NFX & NPTHU & P-value \\
\hline Recipients, $\mathrm{n}$ & 26 & 25 & 42 & \\
Tac + MMF + mPSL & $\mathrm{I} 6(62 \%)$ & $\mathrm{I} 3(52 \%)^{*}$ & $35(84 \%)$ & 0.02 \\
Tac + MMF & 0 & $\mathrm{I}(4 \%)$ & $2(5 \%)$ & 0.54 \\
Tac + Mz + mPSL & $4(15 \%)$ & $4(16 \%)$ & $\mathrm{I}(2 \%)$ & 0.10 \\
CyA + MMF + mPSL & $4(15 \%)$ & $5(20 \%)$ & $\mathrm{I}(5 \%)$ & 0.14 \\
CyA + MMF & $\mathrm{I}(4 \%)$ & $\mathrm{I}(4 \%)$ & $\mathrm{I}(2 \%)$ & $0.9 \mathrm{I}$ \\
CyA + Mz + mPSL & $\mathrm{I}(4 \%)$ & $\mathrm{I}(4 \%)$ & 0 & 0.43 \\
CyA + Mz & 0 & 0 & $\mathrm{I}(2 \%)$ & 0.56 \\
\hline
\end{tabular}

Notes: Values are shown as $\mathrm{n}(\%) . * \mathrm{p}<0.05$ versus NPTHU.

Abbreviations: FX, febuxostat group; NFX, non-febuxostat group; NPTHU, nonpost-transplant hyperuricemia; Tac, tacrolimus; MMF, mycophenolate mofetil; mPSL, methylprednisolone; CyA, cyclosporine; Mz, mizoribine.
Table 3 Effect of febuxostat on serial serum uric acid concentrations $(\mathrm{mg} / \mathrm{dL})$

\begin{tabular}{llllll}
\hline & Baseline & I month & 3 months & 6 months & I 2 months \\
\hline FN & $8.4 \pm 0.3^{\dagger}$ & $6.2 \pm 0.3^{*}$ & $6.4 \pm 0.3^{*}$ & $6.2 \pm 0.3$ & $6.1 \pm 0.3^{*}$ \\
FC & $7.5 \pm 0.4^{\dagger}$ & $6.6 \pm 0.4$ & $7.3 \pm 0.2$ & $6.4 \pm 0.2$ & $5.9 \pm 0.2^{*}$ \\
OB & $7.7 \pm 0.2^{\dagger}$ & $7.7 \pm 0.3$ & $7.7 \pm 0.3^{\dagger}$ & $7.2 \pm 0.3$ & $7.6 \pm 0.4^{\dagger}$ \\
CT & $6.3 \pm 0.3^{+}$ & $6.9 \pm 0.4$ & $6.4 \pm 0.3$ & $6.4 \pm 0.4$ & $6.4 \pm 0.3$ \\
NPTHU & $5.6 \pm 0.2^{*}$ & $5.8 \pm 0.2^{*}$ & $5.8 \pm 0.2^{*}$ & $5.7 \pm 0.2^{*}$ & $5.6 \pm 0.2^{*}$ \\
\hline
\end{tabular}

Notes: Values are shown as the mean \pm standard deviation. $* P<0.05$ versus $O B$, ${ }^{\dagger} P<0.05$ versus $C T$.

Abbreviations: FN, newly treated with febuxostat; FC, conversion to febuxostat; $\mathrm{OB}$, observation without intervention; $\mathrm{CT}$, continued conventional therapy; NPTHU, non-post-transplant hyperuricemia.

\section{Effects of febuxostat on eGFR and blood pressure}

The effects of febuxostat on eGFR and blood pressure are shown in Figure 2. Although baseline eGFR tended to be lower in the FX group than in the NFX group (Figure 2A), the one-year changes in eGFR were similar (Figure 2B). Treatment with febuxostat did not affect systolic or diastolic blood pressure (Figure 2C and D). None of the 93 patients experienced cardiovascular events or allograft loss during the study period.

\section{Effects of febuxostat on liver function, blood cell count, and tacrolimus concentration}

None of the 26 patients in the FX group discontinued treatment with febuxostat, and none experienced severe adverse effects, such as gout attacks or skin rash. Serum alanine aminotransferase, aspartate aminotransferase, alkaline phosphatase, and total bilirubin concentrations were similar in the FX and NFX + NPTHU groups (Figure 3). The effects of febuxostat on blood cell counts and tacrolimus concentration are shown in Figure 4. Changes in white cell count (Figure 4A), hemoglobin (Figure 4B), and platelets (Figure 4C) were similar in the FX and NFX + NPTHU groups. Although dose-normalized tacrolimus blood concentrations tended to be higher in the FX group than in the NFX + NPUHU group, the difference was not statistically significant (Figure 4D).

\section{Differences in practice patterns between nephrologists and urologists}

Of the 93 stable kidney transplant recipients, 53 were treated by nephrologists, 25 by urologists, and 15 alternately by both. Of the 26 recipients in the FX group, 25 were treated by nephrologists and one by an urologist. Nephrologists were significantly more likely than urologists to start febuxostat 


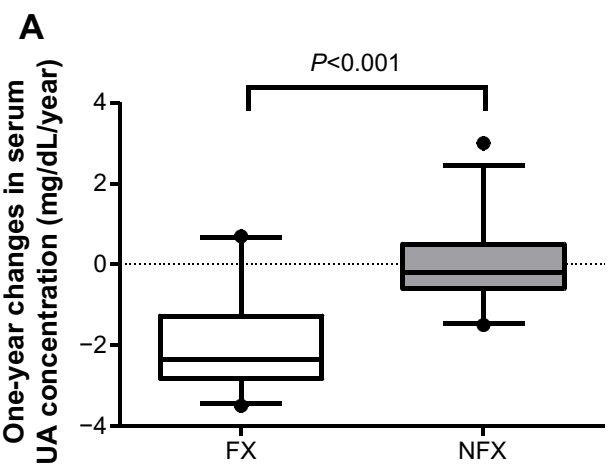

B

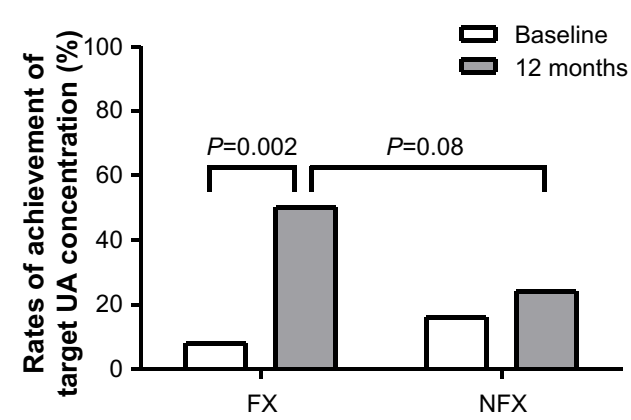

Figure I Effects of febuxostat on (A) one-year changes in serum uric acid and (B) rate of achievement of target uric acid levels. Abbreviations: UA, uric acid; FX, febuxostat group; NFX, non-febuxostat group.

in recipients with PTHU (69\% versus $8 \%$, odds ratio 24.2 , 95\% CI 2.74-214, $P<0.01)$.

\section{Discussion}

This study describes an investigation of the efficacy and safety of febuxostat in stable kidney transplant recipients with PTHU. Treatment of these patients with febuxostat significantly decreased serum uric acid concentrations and increased rates of achievement of target uric acid levels without having severe adverse effects.

A

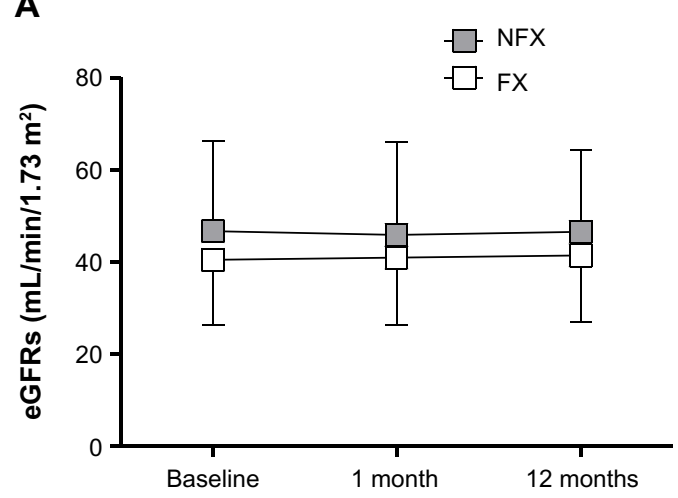

C

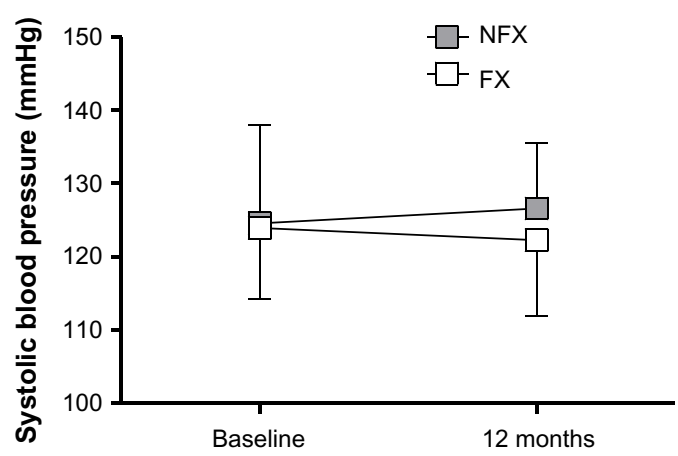

In contrast with our findings for febuxostat, treatment with allopurinol was shown to be unable to reduce serum uric acid levels sufficiently in stable kidney transplant recipients with PTHU. ${ }^{21}$ Patients with chronic kidney disease and hyperuricemia treated with febuxostat had an increased rate of achievement of target uric acid levels..$^{14}$ Our results also indicate that conversion to febuxostat from conventional uric acid-lowering therapy was effective in lowering uric acid levels in kidney transplant recipients who were unable to achieve sufficiently low serum uric acid levels with

B

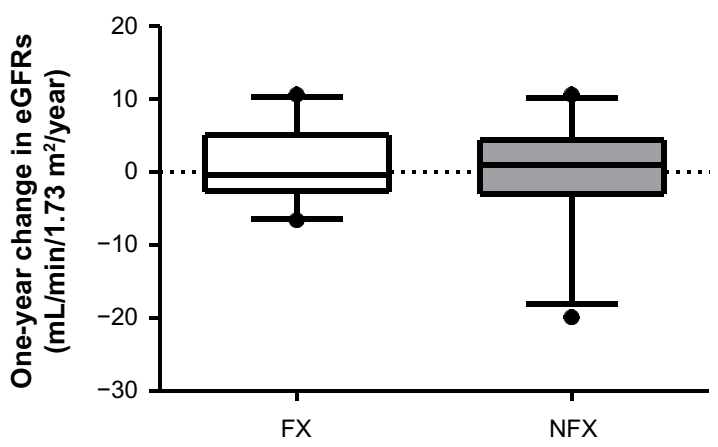

D

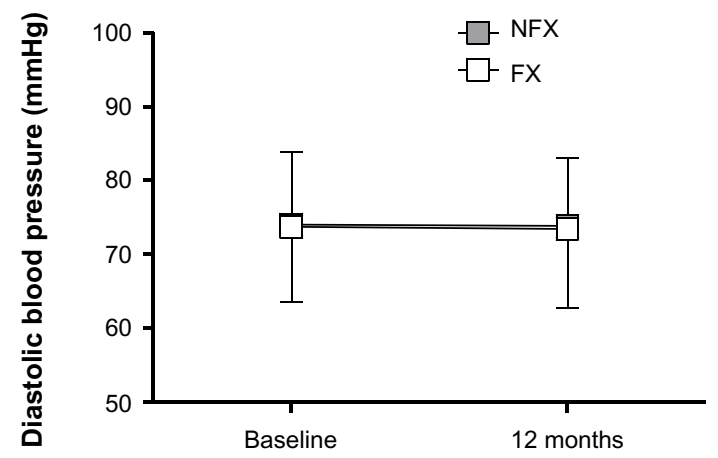

Figure 2 Effects of febuxostat on (A) serial eGFR, (B) changes in eGFR, (C) systolic blood pressure, and (D) diastolic blood pressure. Abbreviations: eGFR, estimated glomerular filtration rate; FX, febuxostat group; NFX, non-febuxostat group. 
A

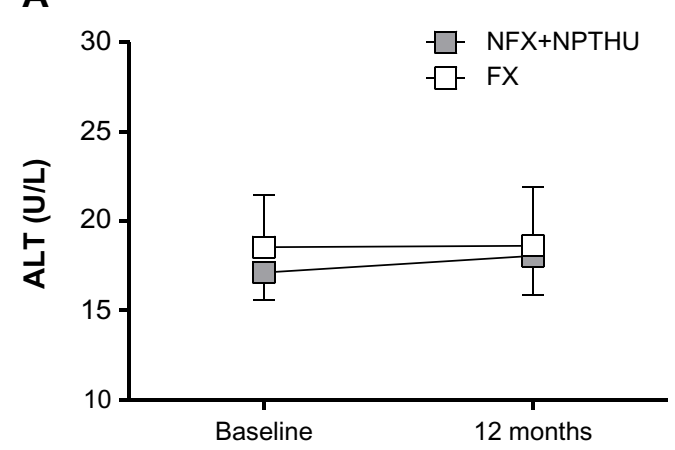

C

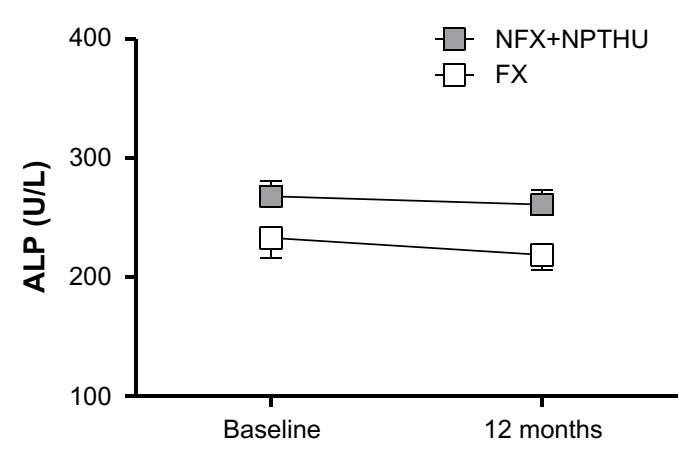

\section{B}

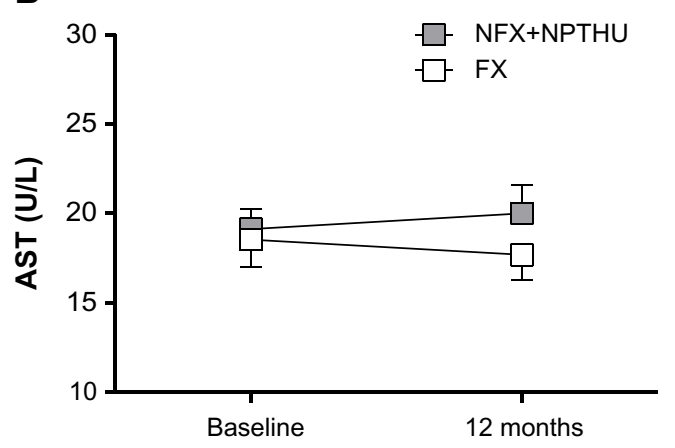

D

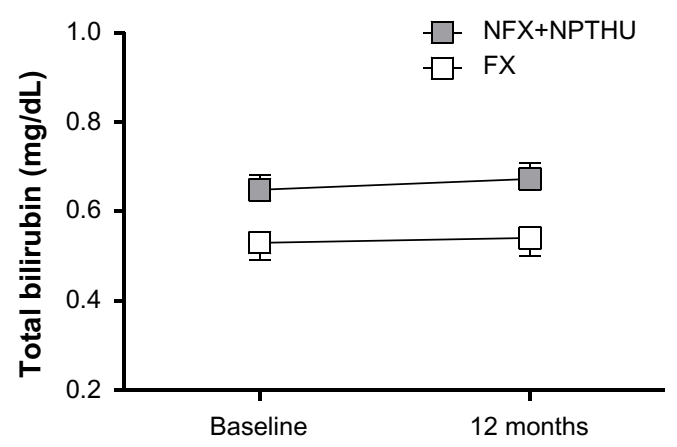

Figure 3 Effects of febuxostat on changes in liver function.

Abbreviations: ALT, alanine aminotransferase; AST, aspartate aminotransferase; ALP, alkaline phosphatase; FX, febuxostat group; NFX, non-febuxostat group; NPTHU, non-post-transplant hyperuricemia.

A

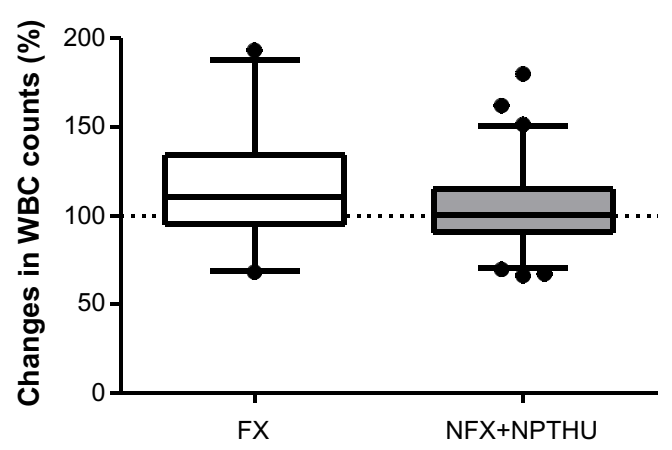

C

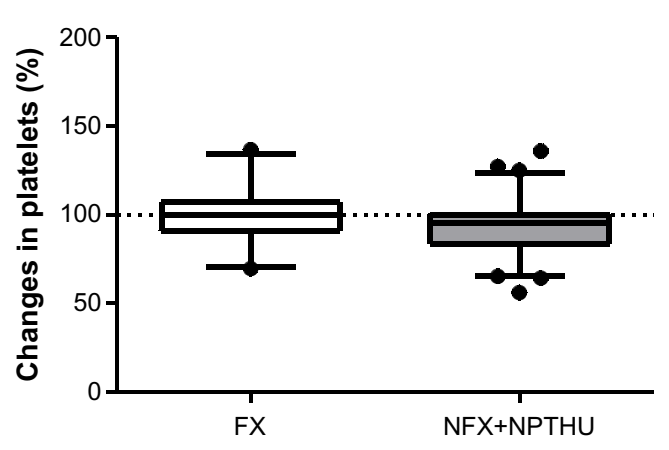

B

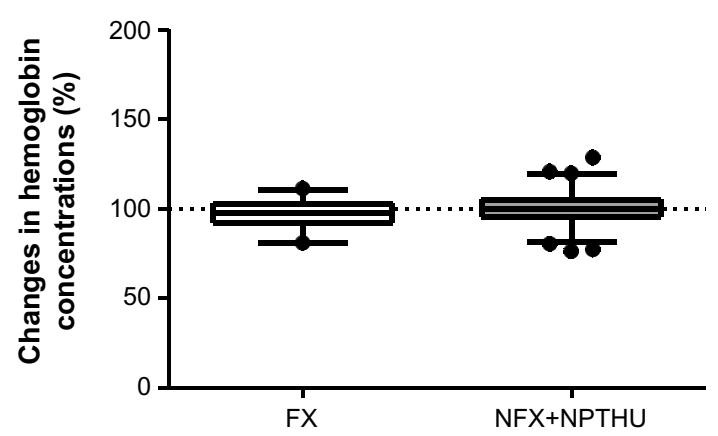

D

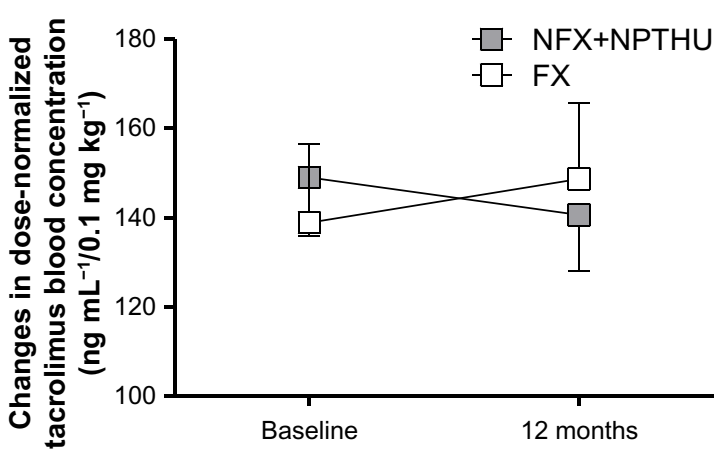

Figure 4 Effects of febuxostat on changes in (A) WBC counts, (B) hemoglobin concentrations, (C) platelet counts, and (D) dose-normalized tacrolimus concentrations. Abbreviations: WBC, white blood cell; FX, febuxostat group; NFX, non-febuxostat group; NPTHU, non-post-transplant hyperuricemia. 
conventional therapy. Because some immunosuppressants cause PTHU in kidney transplant recipients, ${ }^{4,5}$ decreasing immunosuppressant dosages may reduce the risk of PTHU. However, reducing immunosuppressants may increase the risk of acute rejection in kidney transplant recipients. Our results show that febuxostat could significantly decrease serum uric acid concentrations without reducing the doses of immunosuppressants.

It is unclear whether therapeutic interventions in patients with increased uric acid levels are useful in preventing deterioration of kidney function. ${ }^{22-24}$ Moreover, definitions of hyperuricemia differ widely. In the general population, hyperuricemia is generally defined as a uric acid concentration $\geq 7.0 \mathrm{mg} / \mathrm{dL},{ }^{25}$ but detailed information is not available for kidney transplant recipients. Guidelines from the KDIGO (Kidney Disease: Improving Global Outcomes) group recommend that hyperuricemia in kidney transplant recipients be treated when there are complications, such as gout, tophi, or uric acid stones. ${ }^{26}$ In this study, urologists followed this recommendation. Conversely, nephrologists tended to treat asymptomatic PTHU because they knew that hyperuricemia was a risk factor for deterioration of kidney function in patients with chronic kidney disease. Although this study was a retrospective cohort study, the differences in practice patterns between nephrologists and urologists enabled comparisons of interventional and observational treatments for asymptomatic kidney transplant recipients with PTHU.

The differences in practice patterns of nephrologists and urologists are not well researched. Nephrologists have been reported to be more likely to treat anemia and mineral bone disease accompanying chronic kidney disease than non-nephrologists. ${ }^{27}$ Another report showed the effects of practice patterns on allograft outcome in pediatric kidney transplantation. ${ }^{28}$ Neither of these studies, however, described differences in practice patterns between nephrologists and urologists in their treatment of hyperuricemia nor the effects of these differences on allograft prognosis. In general, nephrologists are specialized in the management of blood pressure, proteinuria, anemia, and hyperuricemia, whereas urologists are specialized in surgery and treatment with immunosuppressants. Collaborations between nephrologists and urologists may result in better management of stable kidney transplant recipients.

Febuxostat has been reported to have pleiotropic effects on kidney function other than reducing uric acid. ${ }^{29} \mathrm{Clinical}$ studies have also shown that allopurinol slows the deterioration of kidney function in patients with chronic kidney disease, ${ }^{6,30}$ as well as significantly reducing serum creatinine concentrations in liver transplant recipients with hyperuricemia and renal impairment. ${ }^{31}$ However, no randomized controlled trials have shown that lowering uric acid levels is associated with better allograft survival, allograft function, or recipient survival. Our short-term study showed that lowering uric acid levels was not associated with changes in allograft function or blood pressure. Long-term, large-scale, prospective studies are required to determine whether therapeutic interventions for PTHU can prevent deterioration of allograft function.

No obvious adverse effects were observed in the 26 kidney transplant recipients who received febuxostat. Because febuxostat is a selective xanthine oxidase inhibitor, the combination of febuxostat and azathioprine is not allowed because of their adverse effects on bone marrow. ${ }^{13}$ Recently, azathioprine has been replaced by mycophenolate mofetil or mizoribine as an antimetabolite drug in most kidney transplant recipients. We found no evidence of bone marrow inhibition in any patient who received the combination of febuxostat and mycophenolate mofetil or mizoribine. Tacrolimus concentration has been reported to be associated with broad interindividual pharmacokinetic variability and has been found to be influenced by meals, intestinal edema, ${ }^{20}$ cytochrome $\mathrm{P}$ genotype, ${ }^{32}$ and potential interactions with other drugs, such as proton pump inhibitors and calcium channel blockers. ${ }^{33,34} \mathrm{We}$ found that dose-normalized tacrolimus concentrations tended to be higher in patients who receive febuxostat than in those who did not, although febuxostat does not interact with cytochrome P inhibitors. Additional studies are needed to clarify the effects of febuxostat on tacrolimus concentrations.

This study had several limitations, in particular its small number of patients and its retrospective design. The small sample size of this study may have increased the possibility of type II error. Second, because it was not a randomized controlled study, differences in practice patterns between nephrologists and urologists may have affected our results. Third, baseline eGFR tended to be lower in the FX group than in the NFX group, making it difficult to determine whether lowering of uric acid levels with febuxostat protected allograft function. Finally, the short-term nature of our study precluded determination of the uric acid-lowering effects of febuxostat on cardiovascular events and allograft survival.

In conclusion, our results show that treatment with febuxostat lowers uric acid concentrations sufficiently, without severe adverse effects, in stable kidney transplant recipients 
with PTHU. Further long-term, larger-scale prospective studies are needed to clarify the effects of febuxostat reduction of uric acid levels on allograft survival and cardiovascular events in stable kidney transplant recipients with PTHU.

\section{Acknowledgments}

This work was supported in part by a grant-in-aid for scientific research from the Ministry of Education, Culture, Sports, Science and Technology of Japan (24791653 to TS). We thank Ms Yoshiko Fujita for her technical assistance in the laboratory and Ms Chigusa Nakai for preparation of clinical data.

\section{Disclosure}

The authors declare no conflicts of interest in this work.

\section{References}

1. Abbott KC, Kimmel PL, Dharnidharka V, Oglesby RJ, Agodoa LY, Caillard S. New-onset gout after kidney transplantation: incidence, risk factors and implications. Transplantation. 2005;80(10):1383-1391.

2. Clive DM. Renal transplant-associated hyperuricemia and gout. J Am Soc Nephrol. 2000;11(5):974-979.

3. Numakura K, Satoh S, Tsuchiya N, et al. Hyperuricemia at 1 year after renal transplantation, its prevalence, associated factors, and graft survival. Transplantation. 2012;94(2):145-151.

4. Bahn A, Hagos Y, Reuter S, et al. Identification of a new urate and high affinity nicotinate transporter, hOAT10 (SLC22A13). J Biol Chem. 2008;283(24):16332-16341.

5. Ishikawa I, Maekawa S, Saito T, Horiguchi T, Shinoda A, Ishii H. [Mizoribine-induced hyperuricemia]. Nihon Jinzo Gakkai Shi. 1986;28(10):1353-1357. Japanese.

6. Goicoechea M, de Vinuesa SG, Verdalles U, et al. Effect of allopurinol in chronic kidney disease progression and cardiovascular risk. Clin J Am Soc Nephrol. 2010;5(8):1388-1393.

7. Miao Y, Ottenbros SA, Laverman GD, et al. Effect of a reduction in uric acid on renal outcomes during losartan treatment: a post hoc analysis of the reduction of endpoints in non-insulin-dependent diabetes mellitus with the Angiotensin II Antagonist Losartan Trial. Hypertension. 2011;58(1):2-7.

8. Haririan A, Metireddy M, Cangro C, et al. Association of serum uric acid with graft survival after kidney transplantation: a time-varying analysis. Am J Transplant. 2011;11(9):1943-1950.

9. Min SI, Yun IJ, Kang JM, et al. Moderate-to-severe early-onset hyperuricaemia: a prognostic marker of long-term kidney transplant outcome. Nephrol Dial Transplant. 2009;24(8):2584-2590.

10. Akalin E, Ganeshan SV, Winston J, Muntner P. Hyperuricemia is associated with the development of the composite outcomes of new cardiovascular events and chronic allograft nephropathy. Transplantation. 2008;86(5):652-658.

11. Huang Y, Li YL, Huang H, Wang L, Yuan WM, Li J. Effects of hyperuricemia on renal function of renal transplant recipients: a systematic review and meta-analysis of cohort studies. PLoS One. 2012;7(6): 39457.

12. Day RO, Graham GG, Hicks M, McLachlan AJ, Stocker SL, Williams KM. Clinical pharmacokinetics and pharmacodynamics of allopurinol and oxypurinol. Clin Pharmacokinet. 2007;46(8):623-644.

13. Uloric ${ }^{\circledR}$ Full Prescribing information. Deerfield, IL, USA: Takeda Pharmaceuticals North America, Inc.; 2009.

14. Becker MA, Schumacher HR, Espinoza LR, et al. The urate-lowering efficacy and safety of febuxostat in the treatment of the hyperuricemia of gout: the CONFIRMS trial. Arthritis Res Ther. 2010;12(2):R63.
15. Becker MA, Schumacher HR Jr, Wortmann RL, et al. Febuxostat compared with allopurinol in patients with hyperuricemia and gout. N Engl J Med. 2005;353(23):2450-2461.

16. Zhang W, Doherty M, Pascual E, et al. EULAR evidence based recommendations for gout. Part I: Diagnosis. Report of a task force of the Standing Committee for International Clinical Studies Including Therapeutics (ESCISIT). Ann Rheum Dis. 2006;65(10): 1301-1311.

17. Matsuo S, Imai E, Horio M, et al; Collaborators developing the Japanese equation for estimated GFR. Revised equations for estimated GFR from serum creatinine in Japan. Am J Kidney Dis. 2009;53(6):982-992.

18. Sofue T, Inui M, Kiyomoto $H$, et al. Pre-existing arteriosclerotic intimal thickening in living-donor kidneys reflects allograft function. Am J Nephrol. 2012;36(2):127-135.

19. Sofue T, Inui M, Hara T, et al. Short-term prognosis of living-donor kidney transplantation from hypertensive donors with high-normal albuminuria. Transplantation. 2014;15;97(1):104-110.

20. Sofue T, Inui M, Kiyomoto H, et al. Excess fluid distribution affects tacrolimus absorption in peritoneal dialysis patients. Clin Exp Nephrol. 2013;17(5):743-749.

21. Jacobs F, Mamzer-Bruneel MF, Skhiri H, Thervet E, Legendre C, Kreis H. Safety of the mycophenolate mofetil-allopurinol combination in kidney transplant recipients with gout. Transplantation. 1997;64(7):1087-1088.

22. Chonchol M, Shlipak MG, Katz R, et al. Relationship of uric acid with progression of kidney disease. Am J Kidney Dis. 2007;50(2): 239-247.

23. Kanbay M, Huddam B, Azak A, et al. A randomized study of allopurinol on endothelial function and estimated glomerular filtration rate in asymptomatic hyperuricemic subjects with normal renal function. Clin J Am Soc Nephrol. 2011;6(8):1887-1894.

24. Ito S, Naritomi H, Ogihara T, et al. Impact of serum uric acid on renal function and cardiovascular events in hypertensive patients treated with losartan. Hypertens Res. 2012;35(8):867-873.

25. Mikkelsen WM, Dodge HJ, Valkenburg H. The distribution of serum uric acid values in a population unselected as to tout or hyperuricemia: Tecumseh, Michigan 1959-1960. Am J Med. 1965;39(2):242-251.

26. Kidney Disease: Improving Global Outcomes (KDIGO) Transplant Work Group. KDIGO clinical practice guideline for the care of kidney transplant recipients. Am J Transplant. 2009;9 Suppl 3:S1-S155.

27. Patwardhan MB, Samsa GP, Matchar DB, Haley WE. Advanced chronic kidney disease practice patterns among nephrologists and nonnephrologists: a database analysis. Clin J Am Soc Nephrol. 2007;2(2): 277-283.

28. Van Arendonk KJ, Garonzik Wang JM, Deshpande NA, et al. Practice patterns and outcomes in retransplantation among pediatric kidney transplant recipients. Transplantation. 2013;95(11): 1360-1368.

29. Sanchez-Lozada LG, Tapia E, Soto V, et al. Treatment with the xanthine oxidase inhibitor febuxostat lowers uric acid and alleviates systemic and glomerular hypertension in experimental hyperuricaemia. Nephrol Dial Transplant. 2008;23(4):1179-1185.

30. Siu YP, Leung KT, Tong MK, Kwan TH. Use of allopurinol in slowing the progression of renal disease through its ability to lower serum uric acid level. Am J Kidney Dis. 2006;47(1):51-59.

31. Neal DA, Tom BD, Gimson AE, Gibbs P, Alexander GJ. Hyperuricemia, gout, and renal function after liver transplantation. Transplantation. 2001;72(10):1689-1691.

32. Tsuchiya N, Satoh S, Tada H, et al. Influence of CYP3A5 and MDR1 (ABCB1) polymorphisms on the pharmacokinetics of tacrolimus in renal transplant recipients. Transplantation. 2004;78(8):1182-1187.

33. Bekersky I, Dressler D, Mekki Q. Effect of time of meal consumption on bioavailability of a single oral $5 \mathrm{mg}$ tacrolimus dose. J Clin Pharmacol. 2001;41(3):289-297.

34. MacPhee IA, Fredericks S, Tai T, et al. The influence of pharmacogenetics on the time to achieve target tacrolimus concentrations after kidney transplantation. Am J Transplant. 2004;4(6):914-919. 
Drug Design, Development and Therapy

Dovepress

\section{Publish your work in this journal}

Drug Design, Development and Therapy is an international, peerreviewed open-access journal that spans the spectrum of drug design and development through to clinical applications. Clinical outcomes, patient safety, and programs for the development and effective, safe, and sustained use of medicines are a feature of the journal, which

has also been accepted for indexing on PubMed Central. The manuscript management system is completely online and includes a very quick and fair peer-review system, which is all easy to use. Visit $\mathrm{http}: / / \mathrm{www}$.dovepress.com/testimonials.php to read real quotes from published authors.

Submit your manuscript here: http://www.dovepress.com/drug-design-development-and-therapy-journal 